\title{
Direct operando observation of double layer charging and early SEI formation in Li-ion battery electrolytes
}

\author{
Nataliia Mozhzhukhina ${ }^{1}$,Eibar Flores ${ }^{2}$, Robin Lundström ${ }^{1}$, Ville Nystrom ${ }^{1}$, Paul G. Kitz ${ }^{1}$, Kristina Edström ${ }^{1}$, \\ Erik J. Berg ${ }^{1,2}$
}

${ }^{1}$ Department of Chemistry - Ångström Laboratory, Uppsala University, Box 538, SE-751 21 Uppsala, Sweden.

${ }^{2}$ Electrochemistry Laboratory, Paul Scherrer Institut, CH-5232 Villigen PSI, Switzerland

\begin{abstract}
The solid electrolyte interphase (SEI) is one of the most critical, yet least understood, components to guarantee a stable, long-lived and safe operation of the Li-ion cell. Herein, the early stages of SEI formation in a typical commercially-available $\mathrm{LiPF}_{6}$ and organic carbonate based Li-ion electrolyte are explored by operando surface enhanced Raman spectroscopy (SERS), online electrochemical mass spectrometry (OEMS), and electrochemical quartz crystal microbalance (EQCM). The electric doublelayer is directly observed to charge as $\mathrm{Li}^{+}$solvated by EC progressively accumulates at the negatively charged electrode surface. Further negative polarization triggers SEI formation as evidenced by $\mathrm{H}_{2}$ evolution, electrode mass deposition, and expulsion of the electrolyte from the electrode surface. Electrolyte impurities, such as $\mathrm{HF}$ and $\mathrm{H}_{2} \mathrm{O}$, are reduced early and contribute in a multistep electro-/chemical process to an inorganic SEI layer rich in $\mathrm{LiF}$ and $\mathrm{Li}_{2} \mathrm{CO}_{3}$. These results underline the strong influence of trace impurities on the buildup of the SEI layer, and give new insight into the formation mechanism of the multi-layered SEI. The presented study is a model example of how a combination of complementary and highly surface-sensitive operando characterization techniques offer a step forward to understand interfacial phenomenon and SEI formation mechanisms in future Li-ion batteries.
\end{abstract}

Li-ion batteries (LIB) transform today both the transportation sector and energy infrastructure of our societies towards the use of electric vehicles and renewable electricity. Among the various aspects of the LIB technology, the solid electrolyte interphase (SEI) is one of the most critical, yet least understood component. The SEI is known to be a protective layer forming in situ on the negative electrode primarily in the $1^{\text {st }}$ cycle of the Li-ion cell and thereafter stabilizes its operation until the end of life. However, despite more than two decades of intense research activity, the formation mechanism, composition, and morphology of the SEI remain debated and not completely understood ${ }^{1-3}$.

Numerous advanced micro-/spectroscopic characterization techniques have been developed and applied to study the SEI ${ }^{4}$. The technological advancement in the field has recently been immense and provided us with a multitude of insights into the nature and operating mechanism of these electrode layers. Yet, considering the $\mathrm{nm}$ dimensions and multi-component composite structure of the SEI, no characterizing approach is straight forward and much work remains. Particularly the complex multistep electro-/chemical formation process of the SEI raises several major experimental challenges and real time, in situ or operando, based methodologies are imperative. ${ }^{5}$ Fundamental understanding of factors underlining the formation and stability of the SEI promises to provide tailored SEI design and optimization principles, and therefore more stable and safer Liion batteries of the future.

Raman spectroscopy has traditionally been employed to study bulk electrodes, ${ }^{6,7}$ rather than interfacial processes, because of the limited spatial resolution ( 1-10 micrometer with a modern microscope) and the weak Raman signal (only 1 in $10^{7}$ photons is inelastically scattered). In order to overcome these drawbacks, surface enhanced Raman spectroscopy (SERS) has been extensively developed and applied in the past decades ${ }^{8}$. The SERS effect typically relies on the ability of nanostructured metal surfaces (mainly $\mathrm{Ag}, \mathrm{Au}$ and $\mathrm{Cu}$ ) to enhance signal intensity from near-surface molecules (up to $10^{8}$ times), although the exact physical mechanism behind the enhancement is still under debate ${ }^{8,9}$. SERS has in recent years been applied to investigate battery electrochemistry in various configurations, such as "classical" SERS ${ }^{10,11}$, tip enhanced Raman spectroscopy

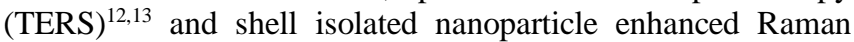
spectroscopy (SHINERS) $)^{14,15}$. "Classical" SERS configuration was employed by Hardwick group to study the mechanism of oxygen reduction in non-aqueous electrolytes using roughened Au electrodes ${ }^{10}$. Nanda et al. employed TERS to study both chemical composition and topography of the SEI on Si as a function of cycle number ${ }^{12}$. Yang et al. employed "classical" (or nanogap) SERS to study electrolyte solvation structures at the electrode/electrolyte interface ${ }^{16,17}$. Particularly the $\mathrm{Li}^{+}$solvation in the common Li-ion solvents EC and DEC at different ionic and solvent fractions was investigated and a deviating $\mathrm{Li}^{+}$ solvation structure at the solid/liquid interface compared to the electrolyte bulk was found. The same authors did however not study those effects under applied potential.

Herein we explore the initial stages of double-layer charging and SEI formation in a typical carbonate $\mathrm{LiPF}_{6}$ based battery electrolyte on a model Au SERS substrate. Interpretation of the results is supported by both operando gas analysis and the measurement of interphase mass deposition on a model $\mathrm{Au}$ electrode substrate by EQCM. The aim of this simplified model system is to disentangle often competing electrochemical reactions governing electrode interphase formation and to provide fundamental understanding that can be extrapolated to practical systems. 

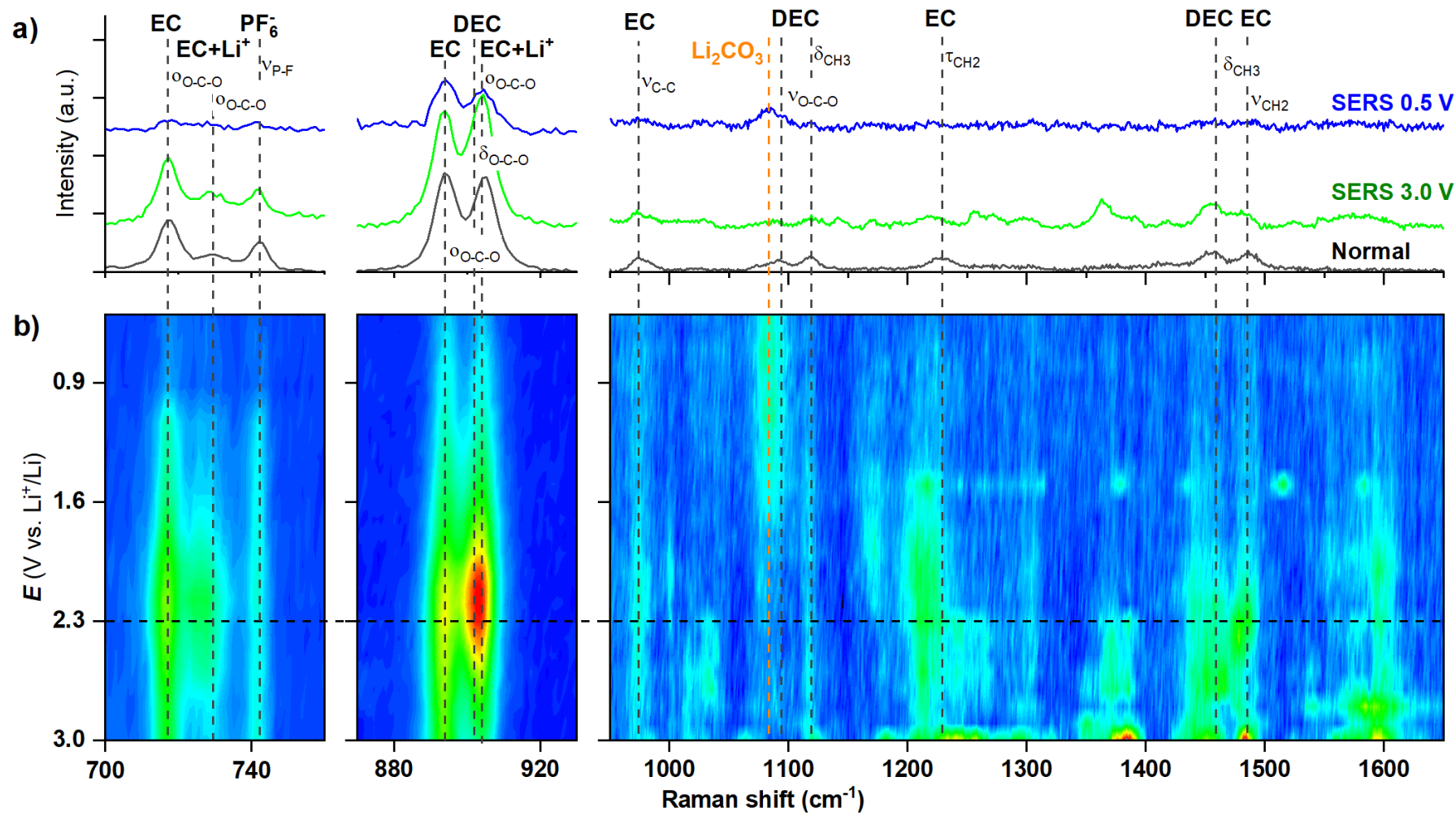

Figure 1. a) Non-enhanced normal Raman spectrum and SERS spectra (at OCP 3.0V and end of sweep 0.5V) of the LP40 electrolyte. b) Operando SERS performed on nanostructured gold electrodes in LP40 electrolyte during potentiostatic steps $(100 \mathrm{mV}, 3.0$ to $0.5 \mathrm{~V})$

Figure 1a shows the non-resonant (normal) and surface enhanced (SERS) Raman spectra of LP40 (1M LiPF 6 in EC:DEC 1:1) as recorded in the operando Raman cell (see S1. Experimental details in SI). Both spectra display essentially the same characteristic peaks stemming from the EC, DEC, and $\mathrm{PF}_{6}^{-}$ electrolyte components (marked by dashed lines, c.f. S2 in SI for details). ${ }^{16,18-20}$ The EC vibrational bands are assigned to ring breathing (oo-C-O), C-C stretching $\left(v_{\mathrm{C}-\mathrm{C}}\right), \mathrm{CH}_{2}$ twisting $\left(\tau_{\mathrm{CH} 2}\right)$ and stretching $\left(v_{\mathrm{CH} 2}\right)$ modes. ${ }^{16,18-20}$ DEC is identified by the O-C-O bending $\left(\delta_{\mathrm{O}-\mathrm{C}-\mathrm{O}}\right)$, symmetric stretching $\left(v_{\mathrm{O}-\mathrm{C}-\mathrm{O}}\right), \mathrm{CH}_{3}$ bond rocking and bending $\left(\delta_{\mathrm{CH} 3}\right)$ bands, while the $\mathrm{PF}_{6}{ }^{-}$anion is here represented by the symmetric stretching of the P-F bond. ${ }^{21}$ Compared to the non-enhanced approach, the SERS spectrum displays several additional bands, primarily in the $1200-1600 \mathrm{~cm}^{-}$ ${ }^{1}$ range, which we relate to vibrational modes either associated with electrolyte/substrate interaction (e.g. EC/DEC adsorption on $\mathrm{Au}$ ) or more likely to electrolyte impurities (e.g. glycols, etc.) attracted to the Au surface. Nanda et al. have for instance shown that carboxylate RCOOLi type compounds result from electrolyte degradation and appear in the $1500-1600 \mathrm{~cm}^{-1}$ range ${ }^{12}$. In any case, minor concentrations of electrolyte impurities are generally known to interfere with the spectroscopic signal (and electrochemistry) in comparable studies in the fields (e.g. electrocatalysis) ${ }^{8,22,23}$. Most importantly, the two peaks of EC solvated to $\mathrm{Li}^{+}$(at 730 and 903 $\mathrm{cm}^{-1}$ ) labelled $\mathrm{EC}+\mathrm{Li}^{+}$, are more intense relative to the nonsolvated EC (at 717 and $894 \mathrm{~cm}^{-1}$ ) peaks when the spectrum is resonance-enhanced. Yang et al. recently made a similar observation, though for a different SERS substrate, but related the increase in the $\mathrm{EC}+\mathrm{Li}^{+}$peak to the geometric confinement effect of the electrolyte in the hotspot nanogap of their SERS substrate. However, turning to operando SERS shown in Fig. 1b, we clearly observe a potential-dependent growth of the $\mathrm{EC}+\mathrm{Li}^{+}$ peaks during negative polarization of the $\mathrm{Au}$, which can be explained by the charging of the electric double-layer and accumulation of $\mathrm{Li}^{+}$and solvating EC at the SERS substrate surface. The intensities of the respective peaks can be extracted by fitting and Fig. 2a shows that the $\mathrm{EC}+\mathrm{Li}^{+}$clearly increases in intensity, whereas the EC peak remain constant until $\sim 2.3 \mathrm{~V}$.
Based on the ratio of the area of $\mathrm{EC}+\mathrm{Li}^{+}$to $\mathrm{EC}$ peaks, a $\mathrm{Li}^{+}$ concentration at the Au surface can be estimated (according to the relationship derived by Yang et al. ${ }^{16}$ ) and found to increase from the expected $1 \mathrm{M}$ at OCP up to $1.5 \mathrm{M}$ at $2.3 \mathrm{~V} \mathrm{vs}$. $\mathrm{Li}^{+} / \mathrm{Li}$ (see S3 in SI for details). All peaks associated with the electrolyte decrease however in intensity thereafter due to SEI formation. The gradual coverage of the $\mathrm{Au}$ surface with degradation products also affects the $\mathrm{EC}+\mathrm{Li}^{+} / \mathrm{EC}$ peak ratio, which returns to its initial value corresponding to $1 \mathrm{M}$ as the LP40 electrolyte loses contact with the charged electrode surface. To the best of our knowledge, this is the first direct observation of double layer charging and subsequent SEI formation in a Li-ion battery electrolyte.

In order to strengthen our conclusions, complementary operando surface characterization was performed. OEMS and EQCM results are presented in Figure $2 b$ and c, respectively. The SEI formation process is electrochemically initiated, as evidenced by a reduction current concurrent with both an increase in gas evolution and electrode mass deposition. The onset of $\mathrm{H}_{2}$ evolution at $\sim 2.6 \mathrm{~V}$ is consistent with the reduction of hydrogen fluoride impurities in the electrolyte as reported by Strmnik et al. ${ }^{24}$ according to

$$
\mathrm{HF}+\mathrm{e}^{-}+\mathrm{Li}^{+} \rightarrow \mathrm{LiF}+1 / 2 \mathrm{H}_{2}
$$

However, both the Raman signal intensity loss and EQCM mass deposition onset later at about $2.3 \mathrm{~V}$. The lag in deposition could be explained by partial LiF solubility in the electrolyte ${ }^{25}$. If the $\mathrm{H}_{2}$ formed in the $1^{\text {st }}$ evolution maximum $(>1.9 \mathrm{~V})$ is integrated (Fig S4) it would correspond to $\sim 16 \mathrm{ppm}$ HF in the electrolyte (according to reaction (1)), which was also confirmed by measuring $\mathrm{F}^{-}$concentration seperately ${ }^{24}$. LiF is also Ramaninactive, which explains the loss of the electrolyte bands without appearance of any new major species. However, $\mathrm{LiF}$ formation is not the only process as several other minor and intermediate Raman bands reminiscent of organic fragments in the 1000$1050,1230-1430$ and $1500-1650 \mathrm{~cm}^{-1}$ regions appear and vanish during the reduction sweep (Figure 1b). Moreover, the calculated electrode mass change per electrons transferred, known as mass per electron (mpe), values from EQCM clearly deviates from the 
$26 \mathrm{~g} / \mathrm{mol} \mathrm{e}^{-}$as expected for $\mathrm{LiF}$ (Fig. 2c), thus indicating that the reduction current is consumed by other processes. Indeed, a band at $1085 \mathrm{~cm}^{-1}$ characteristic for $\mathrm{Li}_{2} \mathrm{CO}_{3}$ appears below $\sim 1.9 \mathrm{~V}$ (Figure 1). $\mathrm{Li}_{2} \mathrm{CO}_{3}$ has been claimed ${ }^{26,27}$ to form in a multistep process starting with reduction of water impurities in the electrolyte

$$
\mathrm{H}_{2} \mathrm{O}+\mathrm{e}^{-} \rightarrow \mathrm{OH}^{-}+1 / 2 \mathrm{H}_{2}
$$

The hydroxide ions can in turn trigger a chemical ring-opening reaction of EC: ${ }^{28}$

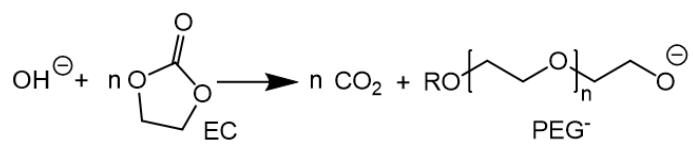

Both products, $\mathrm{CO}_{2}$ and $\mathrm{OH}^{-}$, from reaction (2) and (3) are known to react forming the intermediary hydrocarbonate anion

$$
\mathrm{OH}^{-}+\mathrm{CO}_{2} \rightarrow \mathrm{HCO}_{3}^{-}
$$

which in a final electrochemical step results in lithium carbonate:

$$
\mathrm{HCO}_{3}{ }^{-}+\mathrm{e}+2 \mathrm{Li}^{+} \rightarrow \mathrm{Li}_{2} \mathrm{CO}_{3}+1 / 2 \mathrm{H}_{2}
$$

Although nearly all current is associated with $\mathrm{H}_{2}$ evolution according to OEMS (Fig. 2b), all of reaction (1), (2), and (5) fulfill the criteria of $2 \mathrm{e}^{-} / \mathrm{H}_{2}$ and no discrimination can be made between them on that basis. However, the spectroscopic observation of $\mathrm{Li}_{2} \mathrm{CO}_{3}$ and the concomitant onset of $\mathrm{CO}_{2}$ evolution suggests that water reduction reaction (2) sets in at 1.8 $\mathrm{V}$. If the $\mathrm{H}_{2}$ associated with that process is integrated it would correspond to about $120 \mathrm{ppm}_{2} \mathrm{O}$ in the electrolyte (see $\mathrm{S} 3$ in SI), which is possible considering the difficulty of completely drying several components in the OEMS cell. The band at 1085 $\mathrm{cm}^{-1}$ associated with $\mathrm{Li}_{2} \mathrm{CO}_{3}$ remains however weak, no other band appear, and it may be concluded that the electrode/electrolyte interphase as formed herein is dominated by $\mathrm{LiF}$ and $\mathrm{Li}_{2} \mathrm{CO}_{3}$. No evidence of lithium oxide, peroxide nor lithium hydroxide formation is found (reference Raman spectra of $\mathrm{LiOH}, \mathrm{Li}_{2} \mathrm{O}_{2}, \mathrm{Li}_{2} \mathrm{CO}_{3}$ and $\mathrm{LiF}$ can be found in $\mathrm{S} 2$ of $\mathrm{SI}$ ). These results suggest that the loss of SERS signal is due to the expulsion of the electrolyte from the $\mathrm{Au}$ surface by $\mathrm{LiF}$. Assuming the interphase layer consists predominantly of $\mathrm{LiF}$ with a density of $2.64 \mathrm{~g} / \mathrm{cm}^{3}$, the measured total interphase mass of $0.8 \mu \mathrm{g} / \mathrm{cm}^{2}$ equals the formation of a uniform $\sim 3 \mathrm{~nm}$ thick LiF layer, which would significantly reduce the surfaceenhancement of any electrolyte bands. Below $1 \mathrm{~V}$, a third process slightly deviating from $2 \mathrm{e}^{-} / \mathrm{H}_{2}$ kicks in, but the SERS substrate is already largely passivated as neither $\mathrm{C}_{2} \mathrm{H}_{4}$ nor any other volatile species apart from $\mathrm{H}_{2}$ were observed around $1 \mathrm{~V}$. A very minor evolution of $\mathrm{C}_{2} \mathrm{H}_{4}$ is electrochemically triggered around $2 \mathrm{~V}$, but mechanism will not be further investigated here.

In summary, operando SERS complemented by OEMS and EQCM is shown to provide unique insights into several critical processes governing the performance of electrochemical interphases in Li-ion battery electrolytes. On negative polarization of the model substrate, the double layer is directly observed to charge up to the point where the SEI formation overtakes the spectral response. The electrochemistry is dominated by the reduction of $\mathrm{HF}$ and $\mathrm{H}_{2} \mathrm{O}$ electrolyte impurities as evidenced by $\mathrm{H}_{2}$ evolution and the deposition of the Ramaninactive $\mathrm{LiF}$. $\mathrm{Li}_{2} \mathrm{CO}_{3}$ forms at $\sim 1.8 \mathrm{~V}$ in a multistep electro/chemical process based on $\mathrm{H}_{2} \mathrm{O}$ reduction and $\mathrm{EC}$ decomposition. The presented operando methodology provides powerful means to explore the intricate dynamics of $\mathrm{Li}^{+}$ solvation/coordination, electrolyte/impurity side-reactions, and promises to reveal the formation and operation of the SEI layer itself.

Acknowledgements. E.F. and E.J.B. acknowledge Swiss National Science Foundation (SNSF) under the "Ambizione Energy" funding scheme (Grant 160540). E.J.B, N.M., R.L., V.N. and P.G.K. acknowledge Knut and Alice
Wallenberg(KAW) foundation grant \# is 2017.0204. Base funding from StandUp for Energy is acknowledged.

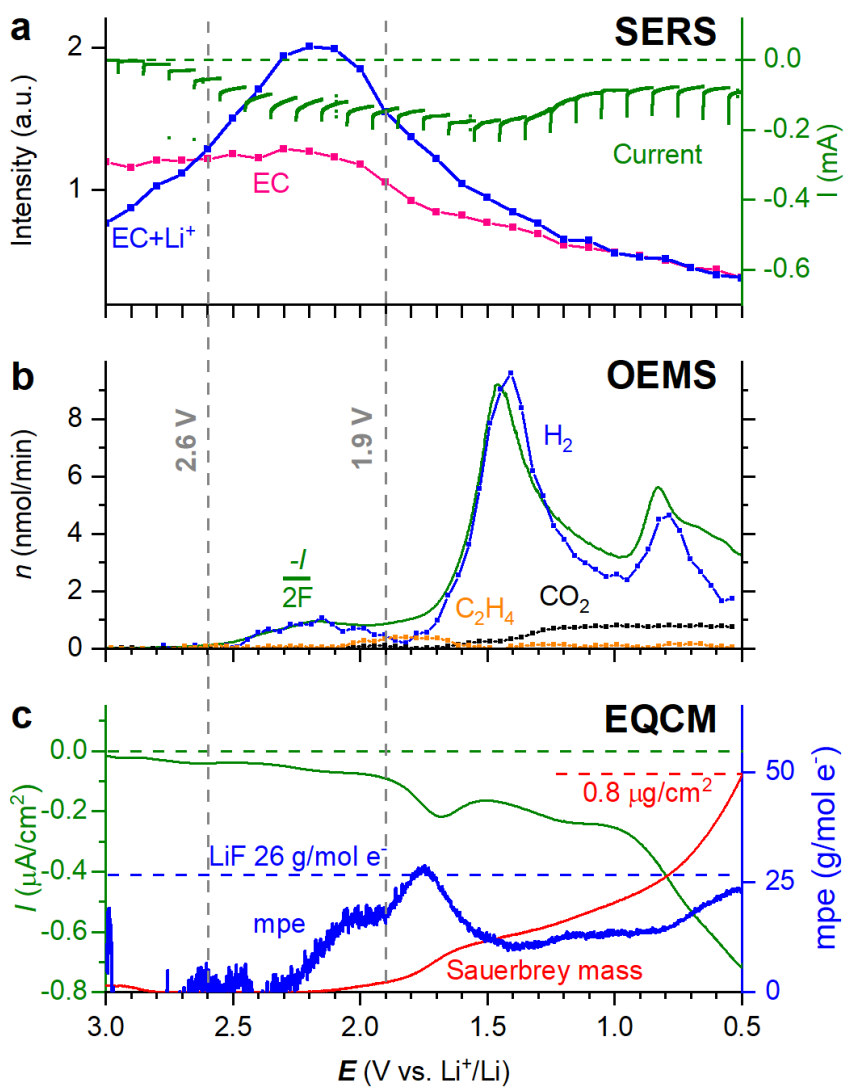

Figure 2. a) The fitted intensities of the Raman peaks at 894 and $904 \mathrm{~cm}^{-1}$, and the current recorded during the potentiostatic steps. b) Faradaic current, $\mathrm{H}_{2}$ and $\mathrm{CO}_{2}$ evolution rates during the reductive sweep. c) Current density, Sauerbrey mass and mass change per mole of electrons transferred (mpe) of the Au-EQCM sensor as function of applied potential.

Supporting information. Experimental details, Raman spectra and assignments of the electrolyte and individual components, OEMS data for $\mathrm{H}_{2}$ evolution and surface $\mathrm{Li}^{+}$concentration calculations can be found in the SI.

\section{References}

(1) Peled, E.; Menkin, S. Review — SEI : Past, Present and Future. 2017, 164 (7). https://doi.org/10.1149/2.1441707jes.

(2) $\mathrm{Xu}, \mathrm{K}$. Electrolytes and Interphases in Li-Ion Batteries and Beyond. 2014. https://doi.org/10.1021/cr500003w.

Winter, B. M. The Solid Electrolyte Interphase - The Most Important and the Least Understood Solid Electrolyte in Rechargeable Li Batteries. 2009, 223, 1395-1406.

Hui, J.; Gossage, Z. T.; Sarbapalli, D.; Herna, K. Advanced Electrochemical Analysis for Energy Storage Interfaces'. 2019. https://doi.org/10.1021/acs.analchem.8b05115.

Edström, K.; Herstedt, M.; Abraham, D. P. A New Look at the Solid Electrolyte Interphase on Graphite Anodes in Li-Ion Batteries. J. Power Sources 2006, 153 (2), 380-384. https://doi.org/https://doi.org/10.1016/j.jpowsour.20 05.05.062. 
(6) M. Julien, C.; Mauger, A. <em>In Situ</Em> Raman Analyses of Electrode Materials for Li-Ion Batteries. AIMS Mater. Sci. 2018, 5 (4), 650-698. https://doi.org/10.3934/matersci.2018.4.650.

(7) Flores, E.; Novák, P.; Berg, E. J. In Situ and Operando Raman Spectroscopy of Layered Transition Metal Oxides for Li-Ion Battery Cathodes. Front. Energy Res. 2018, 6 (August), 1-16. https://doi.org/10.3389/fenrg.2018.00082.

(8) Sharma, B.; Frontiera, R. R.; Henry, A.; Ringe, E.; Duyne, R. P. Van. SERS : Materials, Applications, and the Future Surface Enhanced Raman Spectroscopy ( SERS ) Is a Powerful Vibrational. Mater. Today 2012, 15 (1-2), 16-25. https://doi.org/10.1016/S1369-7021(12)70017-2.

(9) Fleischmann, M.; Hendra, P. J.; McQuillan, A. J. Raman Spectra of Pyridine Adsorbed at a Silver Electrode. Chem. Phys. Lett. 1974, 26 (2), 163-166. https://doi.org/https://doi.org/10.1016/00092614(74)85388-1.

(10) Radjenovic, P. M.; Hardwick, L. J. Time-Resolved SERS Study of the Oxygen Reduction Reaction in Ionic Liquid Electrolytes for Non-Aqueous LithiumOxygen Cells. Faraday Discuss. 2018, 206 (0), 379392. https://doi.org/10.1039/C7FD00170C.

(11) Matsuo, Y.; Kostecki, R.; McLarnon, F. Surface Layer Formation on Thin-Film LiMn[Sub 2]O[Sub 4] Electrodes at Elevated Temperatures. J. Electrochem. $\begin{array}{lllll}\text { Soc. 2001, } & 148 & \text { (7), } & \text { A687. }\end{array}$ https://doi.org/10.1149/1.1373658.

(12) Nanda, J.; Yang, G.; Hou, T.; Veith, G. M.; Sokolov, A. P.; Nanda, J.; Yang, G.; Hou, T.; Voylov, D. N.; Li, X.; et al. Unraveling the Nanoscale Heterogeneity of Solid Electrolyte Interphase Using Tip- Enhanced Raman Spectroscopy Unraveling the Nanoscale Heterogeneity of Solid Electrolyte Interphase Using Tip-Enhanced Raman Spectroscopy. Joule 3 (8), 2001-2019.

https://doi.org/10.1016/j.joule.2019.05.026.

(13) Touzalin, T.; Joiret, S.; Maisonhaute, E.; Lucas, I. T. Capturing Electrochemical Transformations by TipEnhanced Raman Spectroscopy. Curr. Opin. Electrochem. 2017, 6 (1), 46-52. https://doi.org/https://doi.org/10.1016/j.coelec.2017. 10.016 .

(14) Galloway, T. A.; Cabo-Fernandez, L.; Aldous, I. M.; Braga, F.; Hardwick, L. J. Shell Isolated Nanoparticles for Enhanced Raman Spectroscopy Studies in Lithium-Oxygen Cells. Faraday Discuss. 2017, 205, 469-490. https://doi.org/10.1039/c7fd00151g.

(15) Hy, S.; Felix; Chen, Y.-H.; Liu, J.; Rick, J.; Hwang, B.-J. In Situ Surface Enhanced Raman Spectroscopic Studies of Solid Electrolyte Interphase Formation in Lithium Ion Battery Electrodes. J. Power Sources 2014, 256, 324-328. https://doi.org/10.1016/j.jpowsour.2014.01.092.

(16) Yang, G.; Sacci, R. L.; Ivanov, I. N.; Ruther, R. E.; Hays, K. A.; Zhang, Y.; Cao, P.; Veith, G. M.; Dudney, N. J.; Saito, T.; et al. Probing Electrolyte Solvents at Solid / Liquid Interface Using Gap-Mode Surface-Enhanced Raman Spectroscopy. 2019, 166
(2), 178-187. https://doi.org/10.1149/2.0391902jes.

(17) Hallinan, D. T.; Nanda, J. Electrolyte Solvation Structure at Solid-Liquid Interface Probed by Nanogap Surface-Enhanced Raman Spectroscopy. ACS Nano 2018, 12, 10159-10170. https://doi.org/10.1021/acsnano.8b05038.

(18) Fortunato, B.; Mirone, P.; Fini, G. Infrared and Raman Spectra and Vibrational Assignment of Ethylene Carbonate. Spectrochim. Acta Part A Mol. Spectrosc. 1971, 27 (9), 1917-1927. https://doi.org/https://doi.org/10.1016/05848539(71)80245-3.

(19) Klassen, B.; Aroca, R.; Nazri, M.; Nazri, G. A. Raman Spectra and Transport Properties of Lithium Perchlorate in Ethylene Carbonate Based Binary Solvent Systems for Lithium Batteries. J. Phys.

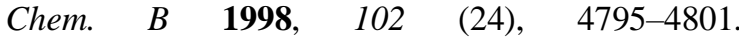
https://doi.org/10.1021/jp973099d.

(20) Allen, J. L.; Borodin, O.; Seo, D. M.; Henderson, W. A. Combined Quantum Chemical/Raman Spectroscopic Analyses of Li+ Cation Solvation: Cyclic Carbonate Solvents - Ethylene Carbonate and Propylene Carbonate. J. Power Sources 2014, 267, 821-830. https://doi.org/10.1016/j.jpowsour.2014.05.107.

(21) Aroca, R.; Nazri, M.; Nazri, G. A.; Camargo, A. J.; Trsic, M. Vibrational Spectra and Ion-Pair Properties of Lithium Hexafluorophosphate in Ethylene Carbonate Based Mixed-Solvent Systems for Lithium Batteries. J. Solution Chem. 2000, 29 (10), 1047-1060.

https://doi.org/10.1023/A:1005151220893.

(22) Garcia-Araez, N.; Rodriguez, P.; Bakker, H. J.; Koper, M. T. M. Effect of the Surface Structure of Gold Electrodes on the Coadsorption of Water and Anions. J. Phys. Chem. C 2012, 116 (7), 4786-4792. https://doi.org/10.1021/jp211782v.

(23) Cabo-Fernandez, L.; Neale, A. R.; Braga, F.; Sazanovich, I. V; Kostecki, R.; Hardwick, L. J. Kerr Gated Raman Spectroscopy of LiPF6 Salt and LiPF6Based Organic Carbonate Electrolyte for Li-Ion Batteries. Phys. Chem. Chem. Phys. 2019, 21 (43), 23833-23842. https://doi.org/10.1039/C9CP02430A.

(24) Strmcnik, D.; Castelli, I. E.; Connell, J. G.; Haering, D.; Zorko, M.; Martins, P.; Lopes, P. P.; Genorio, B.; Østergaard, T.; Gasteiger, H. A.; et al. Electrocatalytic Transformation of HF Impurity to $\mathrm{H}$ 2 and $\mathrm{LiF}$ in Lithium-Ion Batteries. 2018. https://doi.org/10.1038/s41929-018-0047-z.

(25) Jones, J.; Anouti, M.; Caillon-Caravanier, M.; Willmann, P.; Lemordant, D. Thermodynamic of LiF Dissolution in Alkylcarbonates and Some of Their Mixtures with Water. Fluid Phase Equilib. 2009, 285 (1), $62-68$. https://doi.org/https://doi.org/10.1016/j.fluid.2009.0 7.020 .

(26) Kitz, P. G.; Novák, P.; Berg, E. J. Influence of Water Contamination on the SEI Formation in Li-Ion Cells: An Operando EQCM-D Study. ACS Appl. Mater. Interfaces 2020.

https://doi.org/10.1021/acsami.0c01642. 
(27) Metzger, M.; Strehle, B.; Solchenbach, S.; Gasteiger, H. A. Hydrolysis of Ethylene Carbonate with Water and Hydroxide under Battery Operating Conditions. 2016, $163 \quad$ (7), $1219-1225$. https://doi.org/10.1149/2.0411607jes.

(28) Aurbach, D. Nonaqueous Electrochemistry, 1st Editio.; CRC Press: Boca Raton, 1999. https://doi.org/https://doi.org/10.1201/97803678004 99. 